\title{
CrystEngComm
}

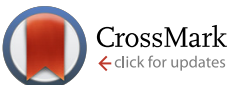

Cite this: CrystEngComm, 2014, 16, 9198

Received 28th May 2014,

Accepted 13th August 2014

DOI: $10.1039 / \mathrm{c} 4 \mathrm{ce} 01095 \mathrm{~g}$

www.rsc.org/crystengcomm

\section{Insight into the mechanism of modulated syntheses: in situ synchrotron diffraction studies on the formation of $\mathrm{Zr}$-fumarate MOF $\dagger$}

\author{
Gesa Zahn,\$ Philip Zerner, Jann Lippke, Fabian L. Kempf, Sebastian Lilienthal, \\ Christian A. Schröder, Andreas M. Schneider and Peter Behrens*
}

\begin{abstract}
In this work, the formation of a $\mathrm{Zr}$-based metal-organic framework (MOF), Zr-fumarate MOF ( $\mathrm{Zr}$-fum MOF), is studied in situ by energy-dispersive diffraction. The $Z r$-fum MOF can be synthesised in DMF as well as in water-based synthesis systems. In both cases, its formation requires modulation, i.e. a monocarboxylic acid which is used as the modulator has to be added to the synthesis mixture. In general, different mechanisms of modulation are possible, for example, deprotonation of the linker molecule (deprotonation modulation) or coordination modulation (wherein the molecules of the modulator compete with the linker molecules for the coordination sites at the inorganic building units). Independently of the specific mechanism, modulation often improves the reproducibility of the MOF synthesis and the crystallinity of the product and may be used to control crystal size and morphology. This study is the first to investigate the kinetics of modulated MOF syntheses with regard to coordination modulation. According to this concept, the addition of a modulator usually decelerates the reaction. Our kinetic investigations show that this is the case for the formation of $\mathrm{Zr}$-fum MOF in the water-based synthesis with formic acid used as a modulator. On the contrary, the addition of formic acid to the DMF-based synthesis results in an accelerating effect. This unexpected effect can be attributed to a small amount of water present in formic acid. Correspondingly, the addition of water to the synthesis mixture also showed an accelerating effect. These investigations emphasise the subtle interplay of the different ingredients in a MOF synthesis. In the case of the $\mathrm{Zr}$-fum MOF, both the modulator formic acid and the water content strongly affect the kinetics of crystallisation. Quantitative evaluation of the kinetic data using the Gualtieri equation provides additional insight into the mechanisms of coordination-modulated MOF formation reactions and excludes the idea of deprotonation modulation.
\end{abstract}

\section{Introduction}

In recent years, metal-organic frameworks (MOFs) or porous coordination polymers (PCPs) have been the focus of intensive research. The porous network and the associated high surface areas as well as the wide variability in chemical functions have demonstrated MOFs as a novel class of fascinating materials for various applications such as gas storage, ${ }^{1}$ separation, ${ }^{2-4}$ catalysis, ${ }^{2-4}$ sensing ${ }^{5}$ and drug delivery systems. ${ }^{6,7}$ Although a large number of MOFs have been synthesised so far, ${ }^{8}$ the crystallisation process has rarely been investigated.

Institut für Anorganische Chemie, Leibniz Universität Hannover, Callinstr. 9, 30167 Hannover, Germany. E-mail: Peter.Behrens@acb.uni-hannover.de, Gesa.Wissmann@acb.uni-hannover.de, Philip.Zerner@acb.uni-hannover.de; Fax: +49511 7623006; Tel: +49511 7623660, +49511 76219177, +495117625187 $\dagger$ Electronic supplementary information (ESI) available: Discussion of data evaluation methods, data of additional kinetic analysis, and powder XRD pattern. See DOI: 10.1039/c4ce01095g

\$ These two authors contributed equally to this study.
Correspondingly, details of the reaction and crystallisation mechanisms have remained rather unexplored, and the conditions for the synthesis of a certain MOF are often determined by undirected explorative work. This lack of knowledge also hampers the preparation of MOFs in certain shapes like nanoparticles, single crystals, or thin films, etc. as well as the fabrication of composites, although this shape control is often important for applications.

The crystallisation behaviour of MOFs depends strongly on the reaction conditions like temperature, concentration of the reactants and the solvent chosen. Another interesting way to influence the outcome of a MOF synthesis is the application of modulation. In such modulated syntheses, a modulating agent - usually a monocarboxylic acid - is added to the reaction mixture. Previous studies on the synthesis of Zr-based MOFs have shown that modulation enhances the reproducibility of the synthesis and can increase the crystallinity of the products. In some cases, modulation allows control of the shape ${ }^{11}$ and the size of the formed crystals, ${ }^{12}$ 
ranging from nanoparticles to single crystals. ${ }^{13}$ It is worth noting that some Zr-based MOFs we have prepared are only accessible when modulation is applied..$^{9,14,15}$

In general, different modulation mechanisms are conceivable. In deprotonation modulation, the modulator enhances the deprotonation of linker molecules (i.e. it acts as a base), thus facilitating the attachment of linker molecules to the inorganic building units (IBUs); consequently, the formation of a MOF framework is accelerated. This was shown to be the case in the synthesis of ZIF-8 with formic acid as the modulator. ${ }^{22}$ In coordination modulation, the modulator competes with the linker molecules for the coordination sites at the IBUs as proposed in the first applications of modulated syntheses. $^{11,12}$ In this way, the formation of amorphous precursors or zirconia gel in the beginning of the reaction is avoided. Nucleation and growth can then occur from dissolved species but proceed at a reduced rate due to the necessary exchange of the coordinated modulator with bridging linker molecules, i.e. reaction rates are decreased. Correspondingly, also the number of nuclei formed is small; these can grow to larger crystals, explaining the size control.

As the only prior kinetic study on modulated MOF syntheses ${ }^{22}$ revealed a deprotonation modulation mechanism, we have performed the present study on the kinetics of the crystallisation of the Zr-fumarate MOF (Zr-fum MOF). This MOF can be synthesised in different solvent systems, namely, in $\mathrm{DMF}^{9}$ and in water. ${ }^{10}$ In both cases, it is necessary to apply the so-called modulation approach; there were indications in these syntheses that a coordination modulation mechanism is operative. ${ }^{11,12}$

In fact, the expected reduction in the reaction rate was observed qualitatively in the synthesis of different Zr-based MOFs. ${ }^{13,15}$ In contrast, the crystallisation of the Zr-fum MOF from DMF was surprisingly accelerated when the amount of the modulator formic acid was increased. ${ }^{9}$ Other experiments by our group on the synthesis of Zr-based MOFs have already shown that the addition of water during modulated synthesis can also influence its outcome. ${ }^{9,13}$ Water accelerates the reaction and may even be necessary to obtain a highly crystalline product. ${ }^{13}$ In fact, a certain minimal amount of water is absolutely necessary in order to build the inorganic building unit (IBU), as each of the $\mathrm{Zr}_{6} \mathrm{O}_{4}(\mathrm{OH})_{4}\left(\mathrm{CO}_{2}\right)_{12}$ IBUs, which are typical of many Zr-based MOFs, contains four oxo and four hydroxo ligands.

In order to gain further insight into the mechanisms of modulated MOF syntheses, we have investigated the kinetics of the formation of the Zr-fum MOF, which can, as already noted above, be synthesised in DMF and in water-based solvent systems. Formic acid was chosen as the modulating agent. The kinetics were studied by in situ energy-dispersive $\mathrm{X}$-ray diffraction (EDXRD) employing synchrotron radiation at beamline F3 at HASYLAB facility, DESY (Deutsches Elektronensynchrotron), in Hamburg, Germany.

Quantitative kinetic information is obtained because the intensity of the recorded Bragg reflections is proportional to the amount of solid that exhibits a diffraction phenomenon. ${ }^{16}$
Kinetic evaluations of the so-obtained data are generally performed by applying a certain model. Calculations proposed by Avrami and Erofeev, ${ }^{17-19}$ Sharp and Hancock, ${ }^{20}$ and Gualtieri ${ }^{21}$ are often used to study crystallisation behaviour and growth mechanisms. Here, we have chosen to present kinetic data obtained by the analysis using the Gualtieri method (eqn (1)), which shows that the degree of crystallisation $\alpha(t)$ is dependent on the time $t$, the fitting parameters $a$ and $b$, the rate constant of growth $k_{\mathrm{G}}$ and the dimension of growth $n$.

$$
\alpha(t)=\frac{1}{1+\mathrm{e}^{-(t-a) / b}}\left[1-\mathrm{e}^{-\left(k_{\mathrm{G}} \cdot t\right)^{n}}\right]
$$

In contrast to the Avrami-Erofeev equation and the Sharp-Hancock evaluation method (which were developed for crystallisation processes in the solid state), the Gualtieri model was constructed to describe solution-mediated transformation reactions (i.e. crystallisation of zeolites from aqueous $\mathrm{NaOH})$. It considers both relevant processes, that of nucleation $(1 /(1+\exp (-(t-a) / b))$ term in eqn (1)) and that of crystal growth $\left(\left[1-\exp \left(-\left(k_{\mathrm{G}} \cdot t\right)^{n}\right)\right]\right.$ term), separately. Therefore, this method appears to be especially well suited to describe the crystallisation behaviour of a MOF from a solution. The choice of the preferred evaluation method is discussed in more detail in the $\mathrm{ESI} \uparrow$ Section $\mathrm{S} 1$; there, the results obtained from our data when the Avrami-Erofeev and the Sharp-Hancock methods are used are also given to have better comparison with other kinetic studies on MOF systems.

In the formalism according to Gualtieri, the total number of nuclei (which are of course not directly visible in diffraction experiments) is considered by including the fitting parameters $a$ and $b$ as well as the dimension of growth $n$ and the rate constant of growth $k_{\mathrm{G}}{ }^{21}$ These fitting parameters can define the probability of nucleation $P_{\mathrm{N}}$, which describes the number of nuclei $N$ present in dependence of the time $t$ (eqn (2)), described by a Gaussian distribution, with $a$ as the position of its maximum and $b$ its variance.

$$
P_{\mathrm{N}}=\frac{\mathrm{d} N}{\mathrm{~d} t}=\mathrm{e}^{-\frac{(t-a)^{2}}{2 b^{2}}}
$$

Furthermore, the Gualtieri method differentiates between the rate constant of growth $k_{\mathrm{G}}$ and the rate constant of nucleation $k_{\mathrm{N}}$, which is given as

$$
k_{\mathrm{N}}=\frac{1}{a}
$$

Our investigation is the first kinetic report to study the coordination modulation synthesis of MOFs. Apart from the study on the formation of a zeolitic-imidazolate framework (ZIF) by Cravillon et al. investigating the deprotonation modulation of formate, ${ }^{22}$ other studies have so far only concentrated on investigating the crystallisation of HKUST-1, ${ }^{23}$ Fe-MIL-53, ${ }^{23}$ Mn-MIL-100 (ref. 24) and Al-based MOFs (CAU-1 and CAU-1- $\left.(\mathrm{OH})_{2}\right){ }^{25}$ There is also a study which is the first to 
focus on the formation of a Zr-based MOF, UiO-66. In this study, no modulator was applied, but hydrochloric acid was used as an additive. ${ }^{26}$

Apart from the directly accessible rate constants, the evaluation methods mentioned above may also provide additional information on the reaction mechanism. For example, Ahnfeldt et $a l .^{25}$ investigated the kinetics of Al-MOFs like CAU-1 and CAU-1-(OH $)_{2}$ using conventional and microwave heating. Based on their evaluation of the kinetic data, performed with the Avrami-Erofeev and the Sharp-Hancock formalisms, the authors postulated that the reaction occurs via different crystallisation mechanisms under different heating conditions. Whereas the microwave-heated reaction apparently proceeds via a diffusion-controlled mechanism (Avrami exponent $n_{\mathrm{AE}} \approx 0.6-0.8$ ), conventionally heated reactions seem to proceed via a phase-boundary-controlled reaction (Avrami exponent $n_{\mathrm{AE}} \approx 1.0-1.1$ ). However, the interpretation of the Avrami exponent (for a detailed discussion see the ESI, $\dagger$ Section S1) is not straightforward, ${ }^{27}$ and similar Avrami exponents may be obtained as a result of different mechanisms. ${ }^{28}$ The investigation of the synthesis of MOF-14 by Millange et al. revealed that this reaction is determined by the nucleation rate because the calculated values for $k_{\mathrm{N}}$ were, in all cases, smaller than values for $k_{\mathrm{G}} \cdot{ }^{29}$ In another study by Millange et al., the authors could identify a metastable intermediate in the crystallisation of MIL-53(Fe). ${ }^{23}$ Our previous qualitative observations that water might have an accelerating effect ${ }^{9}$ are substantiated quantitatively in this publication. The crucial role of the water content was also confirmed recently by Ragon et al. who investigated the crystallisation behaviour of the Zr-based MOF UiO-66, including the addition of hydrochloric acid. ${ }^{26}$

\section{Experimental}

\subsection{Time-resolved in situ X-ray diffraction experiments}

Time-resolved in situ X-ray diffraction experiments were performed at the (now closed) beamline F3 at HASYLABs storage ring DORIS III at DESY in Hamburg, Germany. The ring was operated with a positron beam energy of $4.45 \mathrm{GeV}$. The beamline received white synchrotron $\mathrm{X}$-ray radiation from a bending magnet with a critical energy of $16 \mathrm{keV}$. A horizontal diffractometer with a heavy load sample stage was installed at the beamline station, equipped with a liquid nitrogen-cooled Ge solid-state detector covering selectable diffraction angles from $0^{\circ}$ to $30^{\circ} 2 \theta$. Collimator diameters were chosen between 200 and $300 \mu \mathrm{m}^{2}$. The detector angle was set to $1.9^{\circ}$ or $2.1^{\circ} 2 \theta$.

Syntheses were carried out in sealed $7 \mathrm{~mL}$ Duran ${ }^{\circledR}$ borosilicate glass tubes $1 \mathrm{~cm}$ in diameter and equipped with magnetic stir bars. These glass tubes were filled with the reaction mixture and then placed into a circulating oil-heater constructed by the group of Prof. Bensch from the University of Kiel. ${ }^{30,31}$ This heater consisted of an aluminium block and was heated by a thermostat via a circular oil flow. Reaction temperatures between $43{ }^{\circ} \mathrm{C}$ and $140{ }^{\circ} \mathrm{C}$ were chosen. The measured temperatures had to remain constant for 5 minutes prior to the start of a reaction. The delay time between the moment the reaction vessel was placed in the oven and the opening of the beam shutter was about 1 minute; during that time, a thermocouple indicated that the oil bath had again attained the reaction temperature. The diffraction spectrum covered an energy range from about 10 to $60 \mathrm{keV}$. The acquisition time for a spectrum was either $30 \mathrm{~s}, 60 \mathrm{~s}$ or $120 \mathrm{~s}$. All reactions delivered the first spectrum after the delay time plus the acquisition time. Reactions were considered complete when the growth of the Bragg reflections of the $\mathrm{Zr}$-fum MOF ended in a distinct saturation level.

Real-time evaluation of the raw data was performed using the programs F3tool and F3tool_extens (versions $0.3 \mathrm{k}$ and 0.04, respectively) by André Rothkirch from HASYLAB. The integration was then performed using F3tool by fitting the peaks to obtain the integral intensities of the $\mathrm{Zr} \mathrm{K \alpha} \alpha_{1}$ and $\mathrm{K} \beta_{1}$ fluorescence lines as well as the 111 and 200 Bragg reflections of the Zr-fum MOF. The intensities of the Bragg reflections were normalised to the mean values of the fluorescence lines, thus accounting for the varying ring current, and then scaled from the mean minimal to the mean maximum intensity to obtain the extent of crystallisation $\alpha(t)$, the so-called crystallisation curves. Induction times $t_{0}$ could be obtained directly from the crystallisation curves and are defined as the time when the Bragg peaks could be observed for the first time. Fitting of the crystallisation curves according to the Avrami-Erofeev and Gualtieri equations was performed using the program OriginPro 8.5 by OriginLab, and all fittings according to the Sharp-Hancock model were performed using Microsoft Excel 2007.

\subsection{Scanning electron microscopy}

Scanning electron microscopy (SEM) images were taken using a JEOL JSM-6700F field-emission instrument with an acceleration voltage of $2 \mathrm{kV}$ and a working distance of $3 \mathrm{~mm}$. All samples were dispersed in ethanol and dropped onto a polished carbon block.

\subsection{Synthesis procedures}

2.3.1 Synthesis of $\mathrm{Zr}$-fumarate MOF in a water-based system. Zr-fumarate MOF was synthesised in a water-based system by dissolving $48.2 \mathrm{mg}$ of $\mathrm{ZrCl}_{4}(0.2 \mathrm{mmol}, 1 \mathrm{eq})$ in $4 \mathrm{~mL}$ of $\mathrm{H}_{2} \mathrm{O}$ (222 mmol, $\left.1074 \mathrm{eq}\right)$ at room temperature. To investigate the influence of the amount of the modulator, various concentrations of formic acid, 30-150 eq, were added. $72.8 \mathrm{mg}$ of fumaric acid ( $0.6 \mathrm{mmol}, 3 \mathrm{eq})$ were supplied as the linker. Due to its low water solubility, the fumaric acid was dispersed in the reaction mixture by shaking for approximately $2 \mathrm{~min}$. All experiments were carried out at $43^{\circ} \mathrm{C}$.

2.3.2 Synthesis of Zr-fumarate MOF in a DMF-based system. $24.1 \mathrm{mg}$ of $\mathrm{ZrCl}_{4}(0.1 \mathrm{mmol}, 1 \mathrm{eq})$ were dissolved in $4 \mathrm{~mL}$ of DMF $(52.0 \mathrm{mmol}, 500 \mathrm{eq})$ at room temperature. In order to investigate the temperature dependence of the kinetics of this system, $0.27 \mathrm{~mL}$ of formic acid $(7.3 \mathrm{mmol}, 70 \mathrm{eq})$ and 
$36.4 \mathrm{mg}$ of fumaric acid $(0.3 \mathrm{mmol}, 3 \mathrm{eq})$ were added and mixed until a clear solution was obtained. Reactions were performed at $100{ }^{\circ} \mathrm{C}, 120{ }^{\circ} \mathrm{C}, 130{ }^{\circ} \mathrm{C}$ and $140{ }^{\circ} \mathrm{C}$.

The influence of the amount of the modulator was tested by adding various concentrations of formic acid, 70-130 eq, and $36.4 \mathrm{mg}$ of fumaric acid $(0.3 \mathrm{mmol}, 3 \mathrm{eq})$. The mixture was stirred until a clear solution was obtained. All experiments were carried out at $120{ }^{\circ} \mathrm{C}$.

Furthermore, the influence of the water content in the DMF-based solvent system was examined by adding various amounts of water (0-70 eq) to a reaction mixture containing $24.1 \mathrm{mg}$ of $\mathrm{ZrCl}_{4}(0.1 \mathrm{mmol}, 1 \mathrm{eq})$, in $4 \mathrm{~mL}$ of DMF $(52.0 \mathrm{mmol}$, $500 \mathrm{eq}), 0.27 \mathrm{~mL}$ of formic acid $(7.3 \mathrm{mmol}, 70 \mathrm{eq})$ and $36.4 \mathrm{mg}$ of fumaric acid $(0.3 \mathrm{mmol}, 3 \mathrm{eq})$. These experiments were carried out at $100{ }^{\circ} \mathrm{C}$.

\section{Results and discussion}

The synthesis in a water-based system was performed by varying the amount of formic acid as the modulator. For synthesis in the DMF-based system, the effects of reaction temperature, the amount of the modulator and the water content were investigated.

In the evaluation of all reactions studied, the intensity evolution of the first intensive 111 Bragg reflection was considered. After a short induction time where no diffracting solid could be detected, the reflections gained intensity until they reached their respective maxima. The ratio of the integrated intensities $I(t)$ and the maximum intensity $I_{\max }$ gives the extent of crystallisation $\alpha(t)$ :

$$
\alpha(t)=\frac{I(t)}{I_{\max }}
$$

When $I(t)$ is equivalent to $I_{\max }$, the end of the reaction is reached. Fig. 1 shows a contour plot (Fig. 1a), a 3D view (Fig. 1b), and a crystallisation curve $\alpha(t)$ (Fig. 1c) derived from a typical data set taken during the formation of a $\mathrm{Zr}$-fum MOF. Measurements were taken every 2 minutes. For the measurement in Fig. 1, it can be deduced that the induction time is about 86 min and that the maximum intensity is reached after approximately three and a half hours. In all syntheses, no other crystalline phases except Zr-fum MOF were observed and the phase purity of the products was subsequently proved by powder X-ray diffraction (PXRD) measurements (ESI† Section S2).

\subsection{Water-based synthesis}

To study the crystallisation behaviour of the Zr-fum MOF synthesised in the water-based system, reactions were performed at $43{ }^{\circ} \mathrm{C}$. Reactions that were carried out at higher temperatures, e.g. at $120^{\circ} \mathrm{C}$, were so fast that no crystallisation curves could be recorded. In these cases the reactions were already finished when the first diffraction patterns were measured around $2 \mathrm{~min}$ after the start of the reaction. Thus, the decrease in the reaction temperature to $43^{\circ} \mathrm{C}$ was appropriate to obtain evaluable measurement data.

To study the influence of the variation of the modulator concentration in a water-based synthesis, reactions were performed at $43{ }^{\circ} \mathrm{C} ; 70,100$ and $150 \mathrm{eq}$ of formic acid were added. With low concentrations of the modulator, the reaction is very fast; the induction times are approximately 10 and $20 \mathrm{~min}$ for 70 and $100 \mathrm{eq}$ of formic acid, respectively. When $150 \mathrm{eq}$ of formic acid are added to the reaction mixture, the induction time increases to $86 \mathrm{~min}$ and the plateau corresponding to the end of the crystallisation process is reached much later (Fig. 2). These results support the prevalence of a coordination modulation mechanism instead of deprotonation modulation, as the latter would result in shorter induction times by increasing the amount of the modulator. ${ }^{22}$ Furthermore, deprotonation modulation should, in any way, play only a minor role due to the very acidic conditions caused by the hydrolysis of the $\mathrm{Zr}$ source. The addition of $\mathrm{ZrCl}_{4}$ decreases the $\mathrm{pH}$ value dramatically to around 1 due to the formation of hydrochloric acid; all acids should be protonated to a very high degree under these conditions. Due to the low $\mathrm{pH}$ value attained after the addition of the $\mathrm{Zr}$ source to the synthesis mixture, no influence of the further addition of the modulating formic acid (or of the linking fumaric acid) on the $\mathrm{pH}$ value was found.
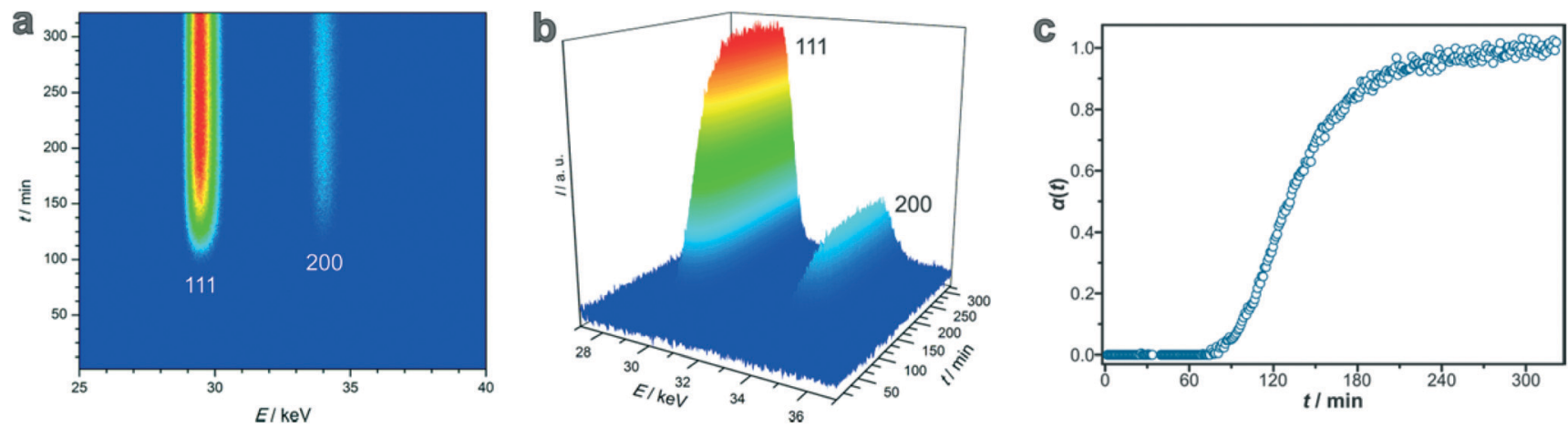

Fig. 1 Examples for the visualisation of time-resolved EDXRD data from $\mathrm{Zr}$-fum MOF synthesis $\left(\mathrm{ZrCl}_{4} / \mathrm{H}_{2}\right.$ fum/formic acid/ $\mathrm{H}_{2} \mathrm{O} \mathrm{molar}$ ratios of $1: 3: 150: 1074$ at $43^{\circ} \mathrm{C}$ ). The 111 and 200 Bragg reflections are visible. (a) 2D contour plot; (b) 3D view; (c) crystallisation curve $\alpha(t)$ of the 111 Bragg reflection. 


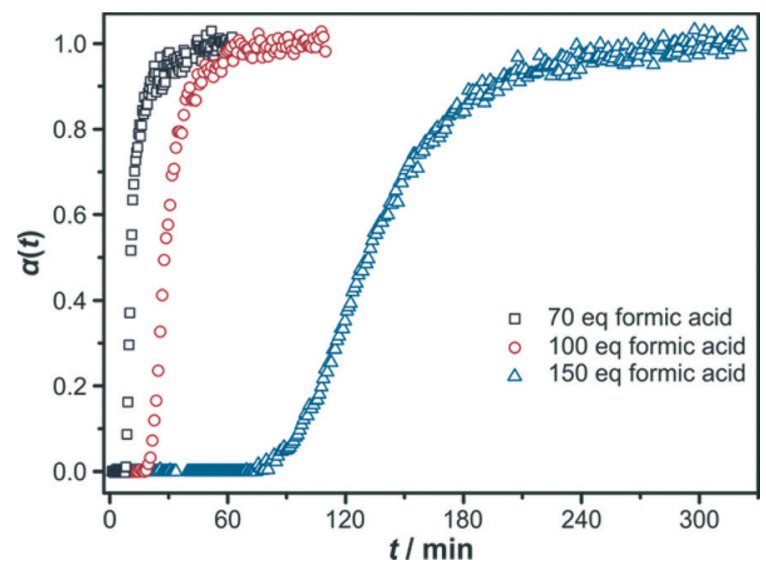

Fig. 2 Extent of crystallisation $\alpha$ plotted against time $t$. Crystallisation curves measured for syntheses of $\mathrm{Zr}$-fum MOF in water-based systems by varying the concentration ( $x$ equivalents) of the modulator formic acid $x\left(\mathrm{ZrCl}_{4} / \mathrm{H}_{2}\right.$ fum/formic acid/water in molar ratios of $1: 3: x: 1074$, $\left.43^{\circ} \mathrm{C}\right)$.

The kinetic data were analysed by applying the Gualtieri method (kinetic data obtained by the evaluation according to the Avrami-Erofeev and the Sharp-Hancock formalisms are presented in the ESI, $\dagger$ Section S1.1). To obtain values of the kinetic parameters, all data of the crystallisation curves were fitted with the Gualtieri equation while keeping $n$ constant at a value of 3 , corresponding to three-dimensional growth. This assumption is justified by the cubic crystal system of the Zr-fum MOF and the observation that the crystal habit is isometric. ${ }^{21}$ An exemplary Gualtieri fitting including the probability of nucleation $P_{\mathrm{N}}$ is shown in Fig. 3; the kinetic parameters obtained for the different reactions are summarised in Table 1.

From these values, it can be concluded that increasing the concentration of the modulator leads to a decrease in the nucleation rate as well as in the growth rate, again substantiating the idea of a coordination modulation mechanism.

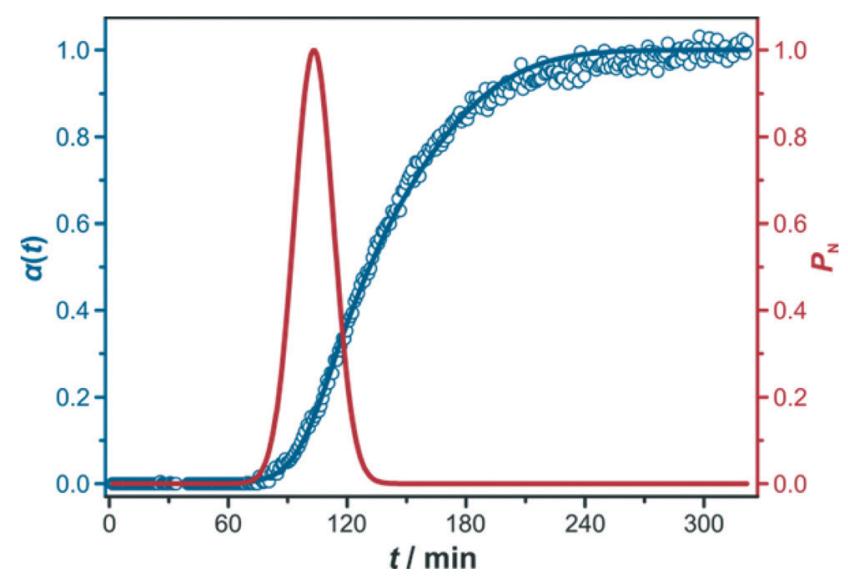

Fig. 3 Extent of crystallisation $\alpha$ plotted against time $t$ (blue circles) and the corresponding Gualtieri fitting (blue curve) as well as the probability for nucleation $P_{\mathrm{N}}$ (red curve). The reaction studied here was carried out at $43{ }^{\circ} \mathrm{C}$ with $\mathrm{ZrCl}_{4} / \mathrm{H}_{2}$ fum/formic acid/ $/ \mathrm{H}_{2} \mathrm{O}$ molar ratios of $1: 3: 150: 1074$.
Table 1 Kinetic parameters obtained by fitting of crystallisation curves with the Gualtieri equation. Crystallisation curves were measured for syntheses of $\mathrm{Zr}$-fum MOF in water-based systems by varying the concentration ( $x$ equivalents) of the modulator formic acid $\left(\mathrm{ZrCl}_{4} / \mathrm{H}_{2}\right.$ fum/formic $\mathrm{acid} /$ water $\left.1: 3: x: 1074,43^{\circ} \mathrm{C}\right)$

\begin{tabular}{lllll}
\hline$x$ & $a / \min$ & $b / \mathrm{min}$ & $k_{\mathrm{G}} / \mathrm{min}^{-1}$ & $k_{\mathrm{N}} / \mathrm{min}^{-1}$ \\
\hline 70 & $13(5)$ & $3(2)$ & $0.10(7)$ & $0.08(5)$ \\
100 & $19(1)$ & $11(1)$ & $0.038(1)$ & $0.053(1)$ \\
150 & $103(1)$ & $10(1)$ & $0.007(1)$ & $0.009(1)$
\end{tabular}

SEM images of the products collected from the syntheses carried out at the synchrotron facility were obtained. Comparing the morphology of the samples prepared with $70 \mathrm{eq}$, $100 \mathrm{eq}$ and $150 \mathrm{eq}$ of formic acid (Fig. 4), it can be observed that the particle size increases with increasing amount of the modulator applied.

These results can be explained by the concept of coordination modulation, which states that both nucleation and growth rates are reduced in the presence of a modulator due to the additional equilibrium involving the exchange of modulator molecules coordinated to the metal cations with linkers. The larger the amount of modulator added to the reaction mixture, the smaller is the nucleation rate $k_{\mathrm{N}}$; therefore, fewer nuclei grow to larger particles, albeit with a decreased growth rate $k_{\mathrm{G}}$, allowing the formation of a highly crystalline material with well-defined particle morphologies and sizes. These insights into the coordination modulation mechanism regarding the formation of particles are in good agreement with previous qualitative observations on the Zr-fum MOF system. ${ }^{9,10}$

\subsection{DMF-based synthesis}

The Zr-fumarate MOF can also be synthesised in a DMFbased system. By varying the temperature of the reaction, the activation energy can be calculated. Furthermore, the effects of the modulator concentration and of the addition of water on the reaction kinetics were investigated.

3.2.1 Variation of the temperature. Zr-fum MOF was synthesised in a temperature range between $100^{\circ} \mathrm{C}$ and $140{ }^{\circ} \mathrm{C}$, using the same synthesis batch composition consisting of $\mathrm{ZrCl}_{4} / \mathrm{H}_{2}$ fum/formic acid/DMF in molar ratios of $1: 3: 70: 500$. Comparing the time dependence of the extent of crystallisation $\alpha(t)$ at different temperatures (Fig. 5), it is obvious that a higher temperature leads to a faster reaction.

At $100{ }^{\circ} \mathrm{C}$, the reaction takes about 7 hours to reach complete crystallisation, whereas at $140{ }^{\circ} \mathrm{C}$ the reaction is completed after only 1 hour. Furthermore, the induction time is significantly decreased by increasing the reaction temperature. The kinetic data were analysed by applying the Gualtieri method (results of Avrami-Erofeev and the Sharp-Hancock evaluations are given in the ESI, $\uparrow$ Section S1.2) and are summarised in Table 2 .

The calculated $k$ values affirm the previous statement. By increasing the temperature, both $k$ values increase as well. Gualtieri's differentiation between the rate constant of 

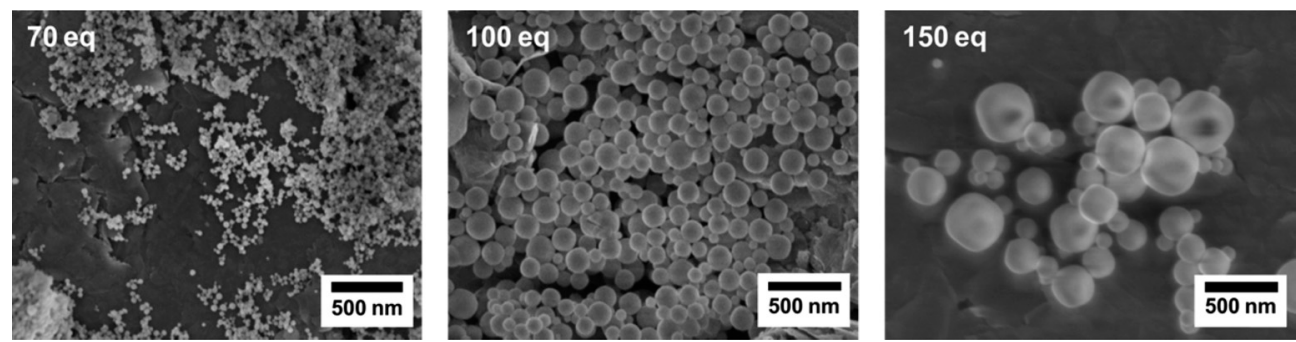

Fig. $4 \mathrm{SEM}$ images of $\mathrm{Zr}$-fum MOF samples synthesised with various concentrations of the modulator (as given in the images) in a water-based synthesis at $43{ }^{\circ} \mathrm{C}\left(\mathrm{ZrCl}_{4} / \mathrm{H}_{2}\right.$ fum/formic acid/ $\left.\mathrm{H}_{2} \mathrm{O} 1: 3: x: 1074\right)$.

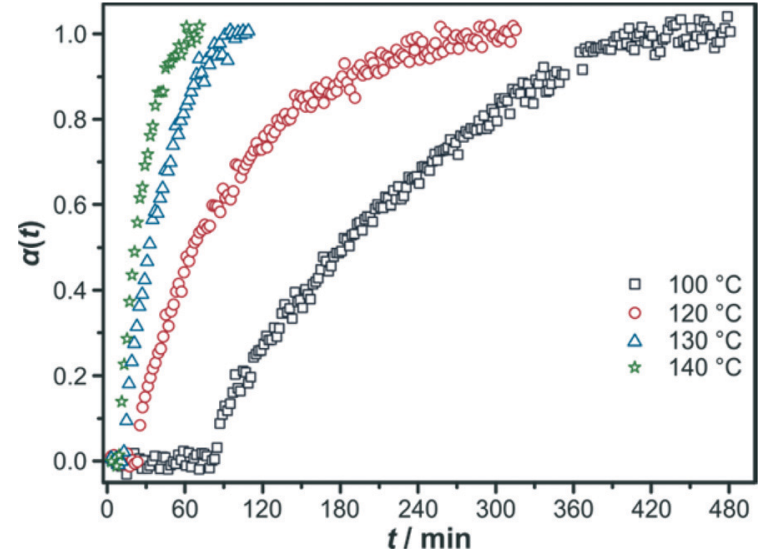

Fig. 5 Extent of crystallisation $\alpha$ plotted against time $t$. Crystallisation curves measured for syntheses of Zr-fum MOF in DMF-based systems by varying the temperature $T\left(\mathrm{ZrCl}_{4} / \mathrm{H}_{2}\right.$ fum/formic acid/DMF in molar ratios of $1: 3: 70: 500)$.

Table 2 Kinetic parameters obtained by fitting of crystallisation curves with the Gualtieri equation. Crystallisation curves were measured for syntheses of $\mathrm{Zr}$-fum MOF in DMF-based systems by varying the temperature $T\left(\mathrm{ZrCl}_{4} / \mathrm{H}_{2}\right.$ fum/formic acid/DMF $\left.1: 3: 70: 500\right)$

\begin{tabular}{lllll}
\hline$T /{ }^{\circ} \mathrm{C}$ & $a / \min$ & $b / \min$ & $k_{\mathrm{G}} / \mathrm{min}^{-1}$ & $k_{\mathrm{N}} / \mathrm{min}^{-1}$ \\
\hline 100 & $183(1)$ & $72(1)$ & $0.010(1)$ & $0.005(1)$ \\
120 & $65(1)$ & $53(1)$ & $0.026(1)$ & $0.015(1)$ \\
130 & $33(1)$ & $17(1)$ & $0.055(2)$ & $0.031(1)$ \\
140 & $21(1)$ & $11(1)$ & $0.074(3)$ & $0.048(1)$
\end{tabular}

growth $k_{\mathrm{G}}$ and the rate constant of nucleation $k_{\mathrm{N}}$ illustrates that the time-defining step in this crystallisation process is the formation of nuclei. The calculation of the rate constants $k_{\mathrm{G}}$ and $k_{\mathrm{N}}$ at different temperatures $T$ offers the opportunity to calculate the activation energies $E_{\mathrm{A}}$. With the help of the corresponding Arrhenius equations (eqn (5) and (6))

$$
\begin{gathered}
k_{\mathrm{G}}=A \mathrm{e}^{-\frac{E_{\mathrm{A}}(\mathrm{G})}{R \cdot T}} ; k_{\mathrm{N}}=A \mathrm{e}^{-\frac{E_{\mathrm{A}}(\mathrm{N})}{R \cdot T}} \\
\ln k_{\mathrm{G}}=\ln A-\frac{E_{\mathrm{A}}(\mathrm{G})}{R T} ; \ln k_{\mathrm{N}}=\ln A-\frac{E_{\mathrm{A}}(\mathrm{N})}{R T}
\end{gathered}
$$

with the gas constant $R$ and $A$ being the pre-exponential factor, the activation energies of nucleation and growth can be determined. The corresponding Arrhenius plots are shown in Fig. 6. The slopes of the linear regressions give the activation energies. The values are $71 \pm 3 \mathrm{~kJ} \mathrm{~mol}^{-1}$ for $E_{\mathrm{A}}(\mathrm{N})$, the activation energy of nucleation, and $66 \pm 6 \mathrm{~kJ} \mathrm{~mol}^{-1}$ for $E_{\mathrm{A}}(\mathrm{G})$, the activation energy of crystal growth.

The calculated activation energies of nucleation and growth thus do not deviate much from each other, both being around $70 \mathrm{~kJ} \mathrm{~mol}^{-1}$. It is tempting to speculate that both energies reflect the same chemical reaction, namely, the exchange of a modulator ligand on a $\mathrm{Zr}$ atom with a bridging linker, a reaction which should not be significantly influenced by the type of entity on which it occurs, i.e. on a small forming nucleus or on a large growing crystal.

In general, the observed values are in good agreement with the activation energies observed in the crystallisation of other MOFs, like ZIF-8 $\left(69 \mathrm{~kJ} \mathrm{~mol}^{-1}\right.$ for $E_{\mathrm{A}}(\mathrm{N}), 72 \mathrm{~kJ} \mathrm{~mol}^{-1}$ for $\left.E_{\mathrm{A}}(\mathrm{G})\right)^{22}$ and HKUST-1 $\left(72 \mathrm{~kJ} \mathrm{~mol}^{-1}\right.$ for $E_{\mathrm{A}}(\mathrm{N}), 64 \mathrm{~kJ} \mathrm{~mol}^{-1}$ for $\left.E_{\mathrm{A}}(\mathrm{G})\right) .{ }^{29}$ In the case of Zr-fumarate MOF and HKUST-1 formation, nucleation seems to be the rate-limiting step, whereas in the ZIF-8 system, it is apparently crystal growth. MOF-14 (114 kJ mol ${ }^{-1}$ for $E_{\mathrm{A}}(\mathrm{N}), 83 \mathrm{~kJ} \mathrm{~mol}^{-1}$ for $\left.E_{\mathrm{A}}(\mathrm{G})\right)^{29}$ and Mn-MIL-100 $\left(127 \mathrm{~kJ} \mathrm{~mol}^{-1} \text { for } E_{\mathrm{A}}(\mathrm{N}), 99 \mathrm{~kJ} \mathrm{~mol}^{-1} \text { for } E_{\mathrm{A}}(\mathrm{G})\right)^{24}$ show somewhat higher activation energies; in these cases, again nucleation seems to limit the reaction rate. A very

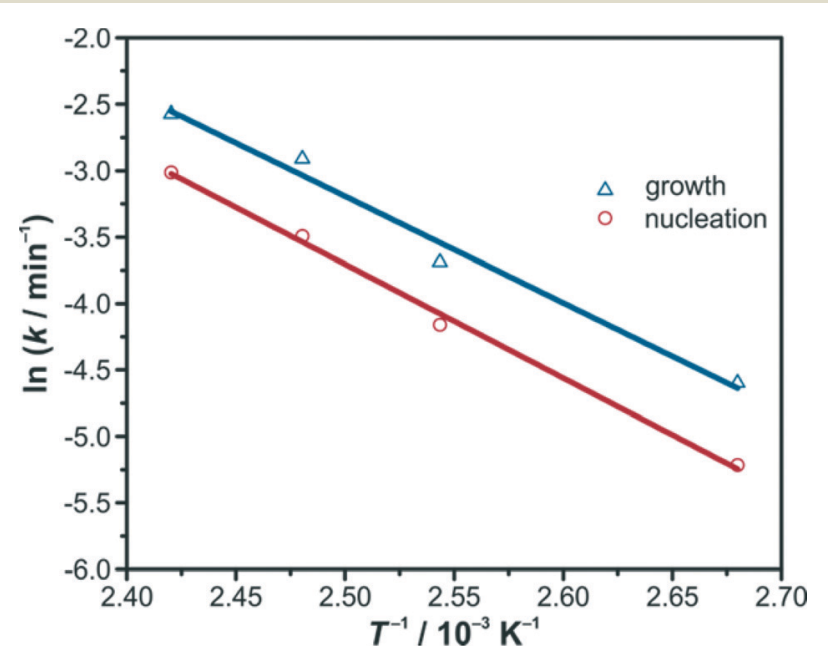

Fig. 6 Arrhenius plots for the temperature-dependent rate constants of growth (blue triangles) and nucleation (red circles) obtained from the Gualtieri evaluation. 
recent study demonstrated that for unmodulated syntheses of the Zr-based MOF UiO-66 the activation energies are much lower. ${ }^{26}$ Depending on the $\mathrm{Zr}$ source used and the amount of hydrochloric acid added to the reaction mixture, the values for $E_{\mathrm{A}}(\mathrm{N})$ vary from $11 \mathrm{~kJ} \mathrm{~mol}^{-1}$ to $39 \mathrm{~kJ} \mathrm{~mol}^{-1}$ and for $E_{\mathrm{A}}(\mathrm{G})$ between $19 \mathrm{~kJ} \mathrm{~mol}^{-1}$ and $46 \mathrm{~kJ} \mathrm{~mol}^{-1}$; all values indicate that growth is the rate-limiting step. Overall, these results strongly suggest that the formation reactions of different MOFs can deliver variable results and strongly depend on the composition of the reaction mixture; comparisons are therefore difficult.

SEM images (Fig. 7) show that the temperature variation only slightly affects the crystal size of the particles. There appears to be a slight increase in the average particle size with increasing temperature, ranging from $c a .100 \mathrm{~nm}$ to ca. $200 \mathrm{~nm}$. As the amount of modulator is the same in all syntheses, the fact only a slight variation of crystallite size is observed is not unexpected. It is accompanied by the disappearance of smaller particles. The SEM images also show that with increasing temperature, the crystallites tend to exhibit more strongly pronounced faces. All these observations are in line with crystal ripening processes, which proceed faster at higher temperatures due to the increased solubility of the particles. The faces formed are (111) faces, obviously the energetically most favourable ones, as $\mathrm{Zr}$-fum MOF crystals typically exhibit octahedral shapes.

3.2.2 Variation of the amount of the modulator. Similarly, reactions were carried out at $120{ }^{\circ} \mathrm{C}$ with variation of the amounts of formic acid as the modulator $\left(\mathrm{ZrCl}_{4} / \mathrm{H}_{2}\right.$ fum $/$ formic acid/DMF 1:3:x:500, $30 \leq x \leq 130$ ). Experiments performed with $30 \mathrm{eq}$ and $50 \mathrm{eq}$ of formic acid result in a precipitation of the solid on the glass tube walls and were therefore not considered for further evaluation. Only the reactions with $70 \mathrm{eq}, 100 \mathrm{eq}$ and $130 \mathrm{eq}$ of the modulator provided reliable results. The influence of the modulator on the time-dependent extent of crystallisation can be seen in Fig. 8 .

The induction time is, in all cases, approximately 20 minutes. Thus, the amount of the modulator does not have an apparent influence on the first precipitation steps in a DMF-based synthesis. This might be an indication of a heterogeneous nucleation process at the glass walls, which is not significantly affected by the modulator. To further rationalise this idea, one has to take into account that the surface chemistry of the glass walls might change by the addition of

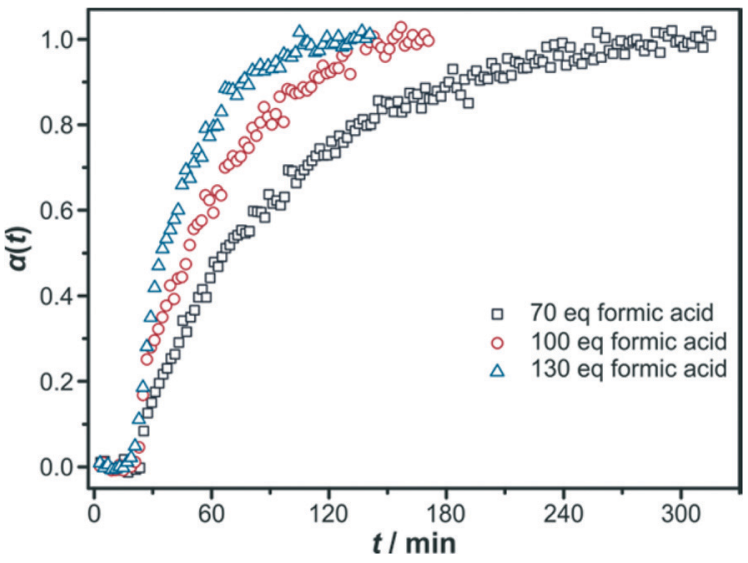

Fig. 8 Extent of crystallisation $\alpha$ plotted against time $t$. Crystallisation curves measured for syntheses of $\mathrm{Zr}$-fum MOF in DMF-based systems by varying the concentration ( $x$ equivalents) of the modulator formic acid $x\left(\mathrm{ZrCl}_{4} / \mathrm{H}_{2}\right.$ fum/formic acid/DMF in molar ratios of $1: 3: x: 500$, $\left.120^{\circ} \mathrm{C}\right)$.

various amounts of the modulator, either by protonation of surface-standing silanol groups or by adsorption of modulator molecules. When only small concentrations of the modulator are present, the glass surface is active and nucleation and crystal growth take place there (as observed when 30 or $50 \mathrm{eq}$ of modulator are added). When the reaction mixture contains $70 \mathrm{eq}$ (or more) of formic acid, nucleation might take place at still active sites on the glass walls; however, crystal growth occurs in the solution.

A surprising fact is observed concerning crystal growth following nucleation: generation of the Zr-fum MOF product is faster and ends earlier the more formic acid is added to the reaction mixture; i.e. the behaviour contradicts the classical action of a modulator as observed in the water-based system and as rationalised by the coordination modulation concept. In contrast to the water-based synthesis (Section 3.1), where the addition of formic acid decelerated the reaction, the addition of formic acid increases the reaction rates in the DMFbased synthesis. This contradictory behaviour has been described before on a qualitative basis. ${ }^{9}$ We ascribe this finding to the accelerating effect of water. Commercially available formic acid contains approximately $2-2.5 \%$ of water as the stabiliser. By increasing the amount of this formic acid in the synthesis, the water content is also concomitantly increased,
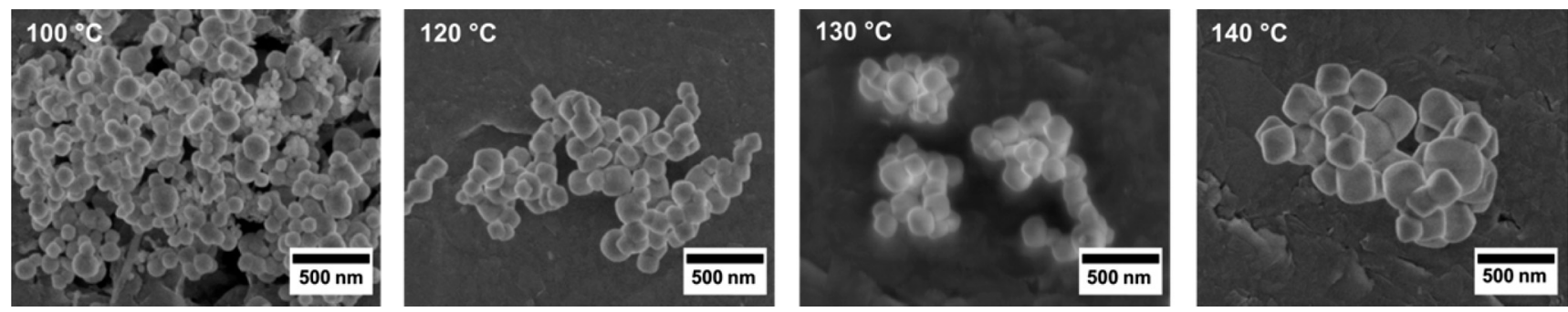

Fig. 7 SEM images of $\mathrm{Zr}$-fumarate MOF synthesised at various temperatures (as given in the images) in a DMF-based synthesis $\left(\mathrm{ZrCl} / 4 / \mathrm{H}_{2}\right.$ fum $/$ formic acid/DMF $1: 3: 70: 500)$. 
explaining the obtained results. The acceleration of the formation of Zr-based MOFs in DMF by the addition of water has been described before ${ }^{9}$ and has recently been quantified by kinetic studies on the formation of UiO-66 (ref. 26) in DMF with various amounts of added water but without any modulator. For the formation of the Zr-fum MOF in DMF, a similar study is presented in Section 3.2.3, substantiating the mentioned hypothesis that adding formic acid accelerates the reaction due to its water content. In water-based synthesis, the water content of the commercially available formic acid does not play a role because water is present in large excess.

The quantitative results, obtained using the Gualtieri evaluation method, of these kinetic results are shown in Table 3 (for the results from Avrami-Erofeev and Sharp-Hancock evaluations, see the ESI, $\dagger$ Section S1.3). It can be seen that the rate constants only slightly increase by increasing the amount of formic acid $x$ from $k \approx 0.01-0.02$ (70 eq of formic acid) to $k \approx 0.03-0.04$ (130 eq of formic acid). This appears reasonable as this is not an effect of the formic acid itself but of the small amounts of concomitantly added water. The differentiation between $k_{\mathrm{G}}$ and $k_{\mathrm{N}}$ reveals that during the formation of the $\mathrm{Zr}$-fumarate MOF under these conditions, nucleation is probably the rate-determining step.

For this system, the kinetic data show that the decelerating action of the modulator molecules is outshone by the water concomitantly introduced by their addition. The strong sensitivity of the reaction to the presence of small amounts of water is not surprising, as the $\mathrm{ZrCl}_{4}$ precursor molecules have to be hydrolysed in order to be able to form the inorganic building units of the MOF structure. A similar sensitivity to the presence of small amounts of water is observed in, for example, sol-gel processes starting from alcoholates and

Table 3 Kinetic parameters obtained by fitting of crystallisation curves with the Gualtieri equation. Crystallisation curves were measured for syntheses of Zr-fum MOF in DMF-based systems by varying the concentration ( $x$ equivalents) of the modulator formic acid $\left(\mathrm{ZrCl}_{4} / \mathrm{H}_{2}\right.$ fum/formic acid/DMF $1: 3: x: 500,120^{\circ} \mathrm{C}$ )

\begin{tabular}{lllll}
\hline$x$ & $a / \mathrm{min}$ & $b / \mathrm{min}$ & $k_{\mathrm{G}} / \mathrm{min}^{-1}$ & $k_{\mathrm{N}} / \mathrm{min}^{-1}$ \\
\hline 70 & $65(1)$ & $53(1)$ & $0.026(1)$ & $0.015(1)$ \\
100 & $46(1)$ & $28(1)$ & $0.035(1)$ & $0.022(1)$ \\
130 & $32(2)$ & $20(1)$ & $0.036(1)$ & $0.031(2)$
\end{tabular}

being carried out in the corresponding alcohol. That the modulator is working nevertheless, in spite of the contradictory kinetic data, becomes clear upon inspection of the SEM pictures of the formed products. These show that the particle sizes increased with increasing amounts of added formic acid (Fig. 9). Taking this into account, a mechanism emerges where the water introduced generally accelerates the reaction by supplying higher quantities of hydrolysed zirconium species. The construction of the MOF framework, however, is still governed by the intermittent coordination of formic acid molecules to the zirconium ions.

3.2.3 Variation of the water content. To confirm the assumption that water introduced together with the formic acid is responsible for the more rapid formation of the Zr-fum MOF with increasing modulator concentration, the influence of the water content on a coordination-modulated synthesis has been investigated independently by in situ EDXRD measurements. For this purpose, reactions were carried out in DMF at constant temperature $\left(100^{\circ} \mathrm{C}\right)$ and at a constant modulator concentration $\left(\mathrm{ZrCl}_{4} / \mathrm{H}_{2}\right.$ fum/formic acid/DMF $1: 3: 70: 500)$. Additionally, a defined amount of water $(0 \leq x \leq 70 \mathrm{eq})$ was added to the reaction mixture to investigate whether the water content influences the reaction rate. As a guideline, the amount of water added with formic acid inevitably corresponds to 3.7 eq of $\mathrm{H}_{2} \mathrm{O}$ when the amount of formic acid is $70 \mathrm{eq}$ and is thus very small under these conditions. The recorded crystallisation curves, as depicted in Fig. 10, indeed show a remarkable effect of the water content.

Without any additional water the precipitation of the resulting product starts after approximately $100 \mathrm{~min}$. The reaction is quite sluggish and finishes after 9 hours. By adding only 5 eq of water, the induction time is more than halved, and by increasing the water content to $30 \mathrm{eq}$ or even 70 eq the induction time is reduced dramatically ( 7 minutes and 3 minutes, respectively), although the modulator is still present in the reaction mixture. Thus, the reaction becomes faster the more water is added. These results can be quantified by analysis with the Gualtieri evaluation (Table 4; for data from Avrami-Erofeev and Sharp-Hancock evaluations see the ESI, $\dagger$ Section S1.4).

All $k$ values strongly increase. By adding 70 eq of water, the rate constants increase by two orders of magnitude compared to synthesis without any additional water. This result
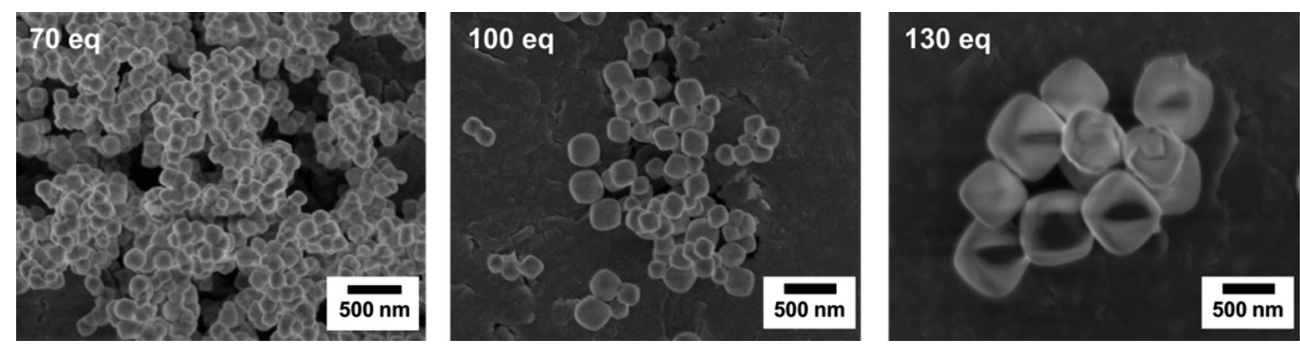

Fig. 9 SEM images obtained from $\mathrm{Zr}$-fumarate MOF synthesised with various amounts of formic acid in a DMF-based synthesis $\left(\mathrm{ZrCl} 4_{4} / \mathrm{H}_{2}\right.$ fum/formic acid/DMF $1: 3: x: 500)$ at $120^{\circ} \mathrm{C}$. 


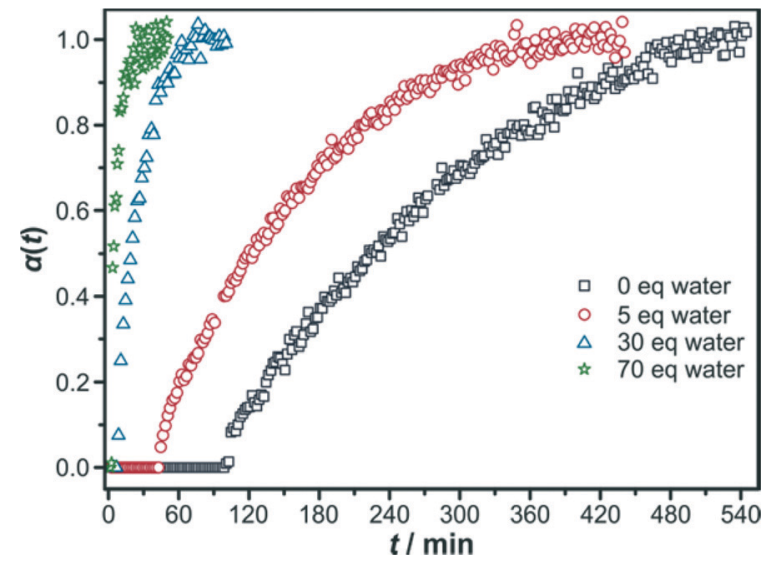

Fig. 10 Extent of crystallisation $\alpha$ plotted against time $t$. Crystallisation curves measured for syntheses of $\mathrm{Zr}$-fum MOF in DMF-based systems by varying the concentration ( $x$ equivalents) of the water content $\left(\mathrm{ZrCl}_{4} / \mathrm{H}_{2}\right.$ fum/formic acid/water/DMF in molar ratios of $1: 3: 70: x: 500$, $\left.100^{\circ} \mathrm{C}\right)$.

Table 4 Kinetic parameters obtained by fitting of crystallisation curves with the Gualtieri equation. Crystallisation curves were measured for syntheses of Zr-fum MOF in DMF-based systems by varying the concentration ( $x$ equivalents) of the water content $\left(\mathrm{ZrCl}_{4} / \mathrm{H}_{2}\right.$ fum/formic acid/water/ DMF $\left.1: 3: 70: x: 500,100{ }^{\circ} \mathrm{C}\right)$

\begin{tabular}{lllll}
\hline$x$ & $a / \min$ & $b / \min$ & $k_{\mathrm{G}} / \mathrm{min}^{-1}$ & $k_{\mathrm{N}} / \mathrm{min}^{-1}$ \\
\hline 0 & $226(1)$ & $89(1)$ & $0.008(1)$ & $0.004(1)$ \\
5 & $123(2)$ & $67(2)$ & $0.016(1)$ & $0.008(1)$ \\
30 & $19(1)$ & $13(1)$ & $0.091(4)$ & $0.053(1)$ \\
70 & $2(1)$ & $6(1)$ & $0.26(2)$ & $0.5(5)$
\end{tabular}

is confirmed by SEM images (Fig. 11). It is found that the water content strongly influences the particle size as well. The particle size becomes smaller the more water is added to the reaction mixture. Without any additional water, the particle size is around $100 \mathrm{~nm}$, whereas it decreases to around $20 \mathrm{~nm}$ when 70 eq of water are used. When the decelerating effect of the modulator is constant, an increase in the water content leads to faster formation of more nuclei. Since the amount of reactants remains constant throughout the experiments, these can grow only to small particles.

All findings concerning the influence of water on the DMF-based modulated synthesis confirm the enormous bearing of water on the crystallisation behaviour. It has become clear that even small amounts of water as introduced by addition of formic acid to an otherwise water-free synthesis mixture has a notable influence on the reaction rate, as described in Section 3.2.2.

\section{Conclusions}

In this work we have investigated the crystallisation behaviour of the Zr-fumarate MOF from a water-based as well as in a DMF-based system in modulated synthesis. By applying energy-dispersive diffraction, the formation of the Zr-fumarate MOF was monitored in situ revealing a coordination modulation mechanism. Concerning the water-based synthesis, the modulating effect of the formic acid led to a deceleration of the reaction while the induction time increased. The higher the amount of the modulator added to the synthesis mixture, the slower the reaction proceeded. This result is in line with the concept of coordination modulation where the molecules of the modulator compete with the linker molecules for the coordination sites at the metal atoms.

On the contrary, in the DMF-based synthesis, the reaction is accelerated by increasing the amount of formic acid added. This effect can be attributed to the water content of the formic acid which is concomitantly added to the reaction mixture. This low water content of the commercially available formic acid becomes crucial when the reaction is performed in an organic solvent. Investigations concerning the influence of the water content in a DMF-based system proved that water has indeed a strong accelerating effect on the formation of the Zr-fumarate MOF. These findings show that MOF syntheses are very sensitive to the actual composition of the reaction batch and to the reaction conditions.

Quantitative evaluation of the measured data according to the Gualtieri equation ${ }^{21}$ gives further insight into the mechanism of modulated reactions. It implies that in most cases the nucleation process is the rate-limiting mechanism, as $k_{\mathrm{N}}<k_{\mathrm{G}}$. When induction times are insensitive to the variation of the reaction conditions, as is the case in the synthesis of Zr-fum MOF in DMF-based systems by varying the modulator concentration, this can be an indication of heterogeneous nucleation, which is indeed directly observed in this system at a small modulator concentration (crystallisation on the glass walls of the container). The finding that the activation energies for nucleation and growth are very similar, as
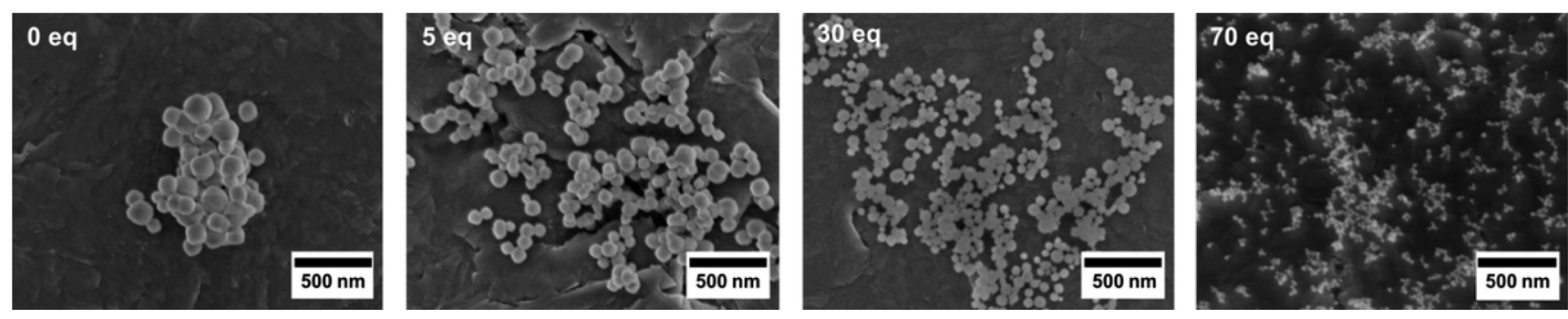

Fig. 11 SEM images obtained from Zr-fumarate MOF synthesised with various amounts of water $x$ added to a DMF-based synthesis $\left(\mathrm{ZrCl} / \mathrm{H}_{2}\right.$ fum/formic $\mathrm{acid} /$ water/DMF $1: 3: 70: x: 500,100{ }^{\circ} \mathrm{C}$ ). 
observed in the study of the reaction in the DMF-based system at variable temperatures, might be due to a coincidence, but does fit well to the concept of coordination modulation, which implies that nucleation and growth proceed via exchange of modulator molecules to linkers. A corresponding temperature-variable study on the reaction taking place in water would be very interesting.

Clearly, more kinetic studies on the formation of MOFs are necessary. Unfortunately, timely access to corresponding high-level experiments at synchrotron beamlines is difficult.

Finally, our investigations suggest that the Gualtieri equation appears to be well suited for the evaluation of kinetic data from reactions where a solid is formed from a liquid solution.

\section{Acknowledgements}

The authors thank DESY for the beamtime approval (project no.: I-20110550), André Rothkirch and Jörn Donges for software and technical support at beamline F3 and Janosch Cravillon, Maria Schweinefuß and Michael Wiebcke for their assistance during the data evaluation and for valuable discussions. We gratefully acknowledge the group of Prof. Bensch from Christian-Albrechts-Universität in Kiel, in particular Nicole Pienack, for providing the oven equipment. This work profited from the DFG priority program 1362 (Porous Metal-Organic Frameworks).

\section{Notes and references}

1 M. Eddaoudi, J. Kim, N. Rosi, D. Vodak, J. Wachter, M. O'Keeffe and O. M. Yaghi, Science, 2002, 295, 469.

2 S. T. Meek, J. A. Greathouse and M. D. Allendorf, Adv. Mater., 2011, 23, 249.

3 H.-L. Jiang and Q. Xu, Chem. Commun., 2011, 47, 3351.

4 A. U. Czaja, N. Trukhan and U. Müller, Chem. Soc. Rev., 2009, 38, 1284.

5 S. Achmann, G. Hagen, J. Kita, I. M. Malkowsky, C. Kiener and R. Moos, Sensors, 2009, 9, 1574.

6 S. Keskin and S. Kizllel, Ind. Eng. Chem. Res., 2011, 50, 1799.

7 P. Horcajada, R. Gref, T. Baati, P. K. Allan, G. Maurin, P. Couvreur, G. Férey, R. E. Morris and C. Serre, Chem. Rev., 2012, 112, 1232.
8 H. Furukawa, K. E. Cordova, M. O'Keeffe and O. M. Yaghi, Science, 2013, 341, 974.

9 G. Wißmann, A. Schaate, S. Lilienthal, I. Bremer, A. M. Schneider and P. Behrens, Microporous Mesoporous Mater., 2012, 152, 64.

10 G. Wißmann, H. A. Schulze, J. Lippke, S. König, U. Sazama, M. Fröba and P. Behrens, Microporous Mesoporous Mater., submitted.

11 T. Tsuruoka, S. Furukawa, Y. Takashima, K. Yoshida, S. Isoda and S. Kitagawa, Angew. Chem., 2009, 121, 4833 (Angew. Chem., Int. Ed., 2009, 48, 4739).

12 S. Diring, S. Furukawa, Y. Takashima, T. Tsuruoka and S. Kitagawa, Chem. Mater., 2010, 22, 4531.

13 A. Schaate, P. Roy, A. Godt, J. Lippke, F. Waltz, M. Wiebcke and P. Behrens, Chem. - Eur. J., 2011, 17, 6643.

14 A. Schaate, P. Roy, T. Preuße, S. J. Lohmeier, A. Godt and P. Behrens, Chem. - Eur. J., 2011, 17, 9320.

15 A. Schaate, S. Dühnen, G. Platz, S. Lilienthal, A. M. Schneider and P. Behrens, Eur. J. Inorg. Chem., 2012, 790.

16 R. I. Walton and D. O'Hare, Chem. Commun., 2000, 2283.

17 M. Avrami, J. Chem. Phys., 1939, 7, 1103.

18 M. Avrami, J. Chem. Phys., 1940, 8, 212.

19 M. Avrami, J. Chem. Phys., 1941, 9, 177.

20 J. D. Hancock and J. H. Sharp, J. Am. Ceram. Soc., 1972, 55, 74.

21 A. F. Gualtieri, Phys. Chem. Miner., 2001, 2, 719.

22 J. Cravillon, C. A. Schröder, H. Bux, A. Rothkirch, J. Caro and M. Wiebcke, CrystEngComm, 2012, 14, 492.

23 F. Millange, M. I. Medina, N. Guillou, G. Férey, K. M. Golden and R. I. Walton, Angew. Chem., Int. Ed., 2010, 49, 763.

24 H. Reinsch and N. Stock, CrystEngComm, 2013, 15, 544.

25 T. Ahnfeldt, J. Moellmer, V. Guillerm, R. Staudt, C. Serre and N. Stock, Chem. - Eur. J., 2011, 17, 6462.

26 F. Ragon, P. Horcajada, H. Chevreau, Y. K. Hwang, U. Lee, S. R. Miller, T. Devic, J. Chang and C. Serre, Inorg. Chem., 2014, 53, 2491.

27 E. E. Finney and R. G. Finke, Chem. Mater., 2009, 21, 4692.

28 T. Pradell, D. Crespo, N. Clavaguera and M. T. Clavaguera-Mora, J. Phys.: Condens. Matter, 1998, 10, 3833.

29 F. Millange, R. El Osta, M. E. Medina and R. I. Walton, CrystEngComm, 2011, 13, 103.

30 L. Engelke, M. Schaefer, M. Schnur and W. Bensch, Chem. Mater., 2001, 13, 1383.

31 N. Pienack, C. Näther and W. Bensch, Eur. J. Inorg. Chem., 2009, 937. 